Article

\title{
Study on the Thermal Properties and Enzymatic Degradability of Chiral Polyamide-Imides Films Based on Amino Acids
}

\author{
Weipeng Liu ${ }^{1,2}$, Fuyan $\mathrm{He}^{1,2}$, Wenke Yang ${ }^{1,2}$, Zhizhou Yang ${ }^{1,2}$, Jinshui Yao ${ }^{1,2, *}$ and \\ Hui Zhao ${ }^{1,2,3}$ \\ 1 School of Materials Science \& Engineering, Qilu University of Technology (Shandong Academy of Sciences), \\ Jinan 250353, China; qlulwp@163.com (W.L.); hefuyan555@163.com (F.H.); wkyang@qlu.edu.cn (W.Y.); \\ yzz@qlu.edu.cn (Z.Y.); zhaoh@uestc.edu.cn (H.Z.) \\ 2 Shandong Provincial Key Laboratory of Processing \& Testing Technology of Glass and Functional Ceramics, \\ Jinan 250353, China \\ 3 Institute of Fundamental Sciences and Frontiers, University of Electronic Sceinces and Technology of China, \\ Chengdu 610054, China \\ * Correspondence: yaojsh@qlu.edu.cn; Tel.: +86-531-89631226
}

Received: 29 December 2018; Accepted: 2 February 2019; Published: 10 February 2019

\begin{abstract}
Six kinds of chiral polyamide-imides (PAI3a-3f) have successfully been synthesized via direct polycondensation. The thermal properties of the materials were evaluated by the gravimetric analysis (TGA) and differential scanning calorimetry (DSC). A thermal test was conducted and showed that the polymers have good thermal stability. The $\mathrm{Tg}$ values were affected by the volume effect of the side groups, the internal plasticizing effect and the isolation effect. Polyamide-imides (PAIs) with L configuration showed higher Tg values than PAIs with D configuration. In the enzymatic degradation experiments of PAI films, the results showed that the structure of amino acids have the greatest influence on the degradation performance of PAIs relative to chirality. The PAI films with simple side groups and L configurations were easier to degrade. The degradation rate of natural chiral PAIs were higher than those of non-natural chiral PAIs. This makes it possible to develop polymer materials with specific degradation rates.
\end{abstract}

Keywords: polyamide-imides (PAIs); degradability; amino acids; glass transition temperature (Tg)

\section{Introduction}

Over the past few decades, polymer-based materials have rapidly developed and are used wildly in all areas. Especially biodegradable polymers, have been used extensively in fields such as food packaging, tissue engineering and biomedical application. They promote societal development and have greatly changed our lives [1-3]. Alongside the performance requirements of materials becoming increasingly strict and the rise of environmental consciousness, biodegradability is already not only a functional need, but also an important environmental requirement [4]. Therefore, more and more biodegradable polymers are starting to become a concern for people, especially a polymer that could control the rate of degradation $[5,6]$. Amino acid-based polyamide-imides (PAIs) are a type of high-performance (HP) materials that combine the thermal resistance and mechanical properties of polyimides, and the easy machinability of PA [7,8], as well as having improved degradation properties [9] and cell compatibility [10]. This is due to the introduction of natural chiral sections, which is obviously of great significance for the development of HP, high-processability, and biodegradable polymer materials. 
The strong chiral preference for amino acids and other essential biomolecules is one of the characteristics of a biological system. Many biological events are greatly influenced by the chirality of molecules [11,12], such as the specific rotational directions of some flowers, and the different tastes or smells of a pair of enantiomers [13]. This inspired the research of introducing chiral effects into biomaterial [14]. Gan et al. modified the chiral surface with NIBC enantiomers and found that on the L surface, the extent of absorption is higher than on the D surface [15]. Reeve et al. proved that proteinase K preferentially degrades (L)-PLA as opposed to (D)-PLA [16]. Mastering chiral signals and translating them into macroscopic properties or functions of materials, will bring great advantages to many applications, particularly for degradable materials [17]. In addition, it was also necessary to elucidate the relations between the chemical structure and degradation properties in order to design and develop a new biodegradable polymeric material.

In this paper, PAIs derived from six amino acids with different configuration were prepared. The PAIs were characterized and subjected to biodegradation experiments in buffer solution (containing proteinase-K). The effects of stereochemical composition on the biodegradation performance are discussed.

\section{Materials and Methods}

\subsection{Materials}

L-alanine, D-alanine, glycine, L-valine, L-leucine, L-norvaline, 4,4'-diaminodiphenyl ether (ODA), triphenyl phosphite (TPP), pyromellitic dianhydride (PMDA), N-methyl-2-pyrrolidone (NMP), pyridine (Py), $\mathrm{CaCl}_{2}, \mathrm{~N}, \mathrm{~N}$-dimethylformamide (DMF) and anhydrous ethanol were analytical grade reagents. All chemicals were used directly without further purification.

\subsection{General Characterization}

Proton nuclear magnetic resonance ${ }^{1} \mathrm{H}$ NMR was carried out on a Bruker AVANCE II $400 \mathrm{MHz}$ spectrometer (Swiss) in deuterated dimethyl sulfoxide (DMSO-d6) as the solvent. Powders were mixed with $\mathrm{KBr}$ powders, and the mixture was pressed into a tablet. The Fourier transform infrared (FTIR) spectra of samples was recorded using a Nicolet iS10 (New Castle, DE, USA) spectrophotometer. Specific rotations were determined with a concentration of $0.2020 \mathrm{~g} / 100 \mathrm{~mL}$ in DMF at $25^{\circ} \mathrm{C}$ by a MCP-200 polarimeter (Anton Par, Graz, Austria). Elemental analyses were recorded using Elementar Vario EL model element analysis equipment (Hamburg, Germany). Thermogravimetric analysis (TGA) on polymers was conducted on a TGA/SDTA851 System (Setaram, Caluire-et-Cuire, France) under a nitrogen $\left(\mathrm{N}_{2}\right)$ atmosphere at a flow rate of $10{ }^{\circ} \mathrm{C} / \mathrm{min}$. The glass transition temperatures $(\mathrm{Tg})$ of the polymers were studied on a differential scanning calorimetry (DSC; Q-100, TA instruments, New Castle, DE, USA) instrument. The molecular weights of polymers were analyzed with gel permeation chromatography (GPC) and multi-angle laser light scattering (MALLS, Dawn Heleos), using a linear MZGel SD Plus GPC column set (two columns, $5 \mu \mathrm{m}$ particles, $300 \times 8 \mathrm{~mm}$ ) with DMF as eluent at room temperature with a flow rate of $1 \mathrm{~mL} / \mathrm{min}$ and a concentration of the polymer of ca. $1 \mathrm{mg} / \mathrm{mL}$. The calibration was based on polystyrene standard. A UV-Vis detector at $\lambda=254 \mathrm{~nm}$ and a IR detector were used for the signal recording. The water contact angles of polymers were characterized in SL200B type contact angle tester (Boston, MA, USA)

\subsection{Synthesis of Diacid Monomers}

Diacid monomers (2a-2f) were obtained by following the method reported in the literature [18] as shown in Scheme 1. Yields and physical properties of the diacid monomers are described in Table S1. 


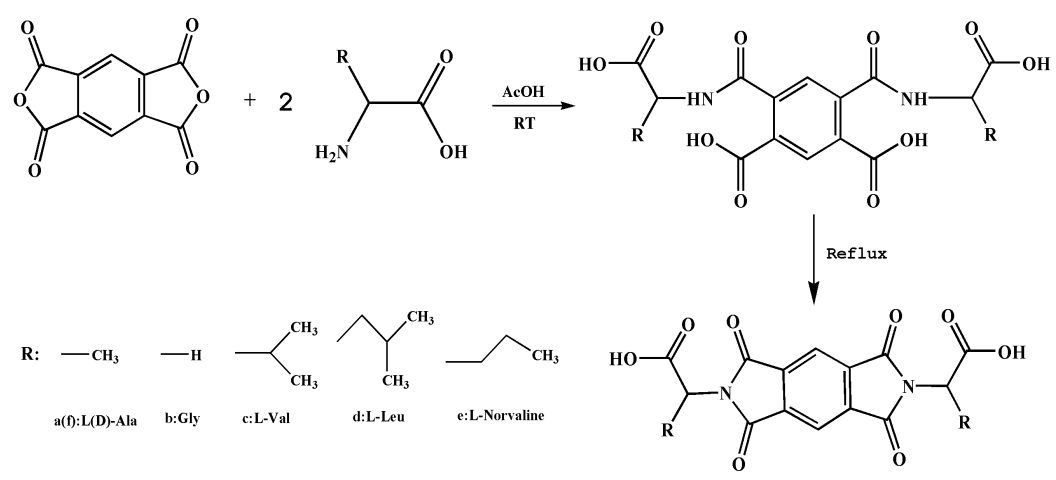

Scheme 1. Polymerization scheme of chiral diacid monomers.

\subsection{Synthesis of Polyamide-Imides}

Polyamide-imides (PAI3a-PAI3f) were synthesized by direct polycondensation of diacid monomers with 4,4'-diaminodiphenyl ether under the reported procedure [19] as shown in Scheme 2. Yields and physical properties of polymers are shown in Table S2.

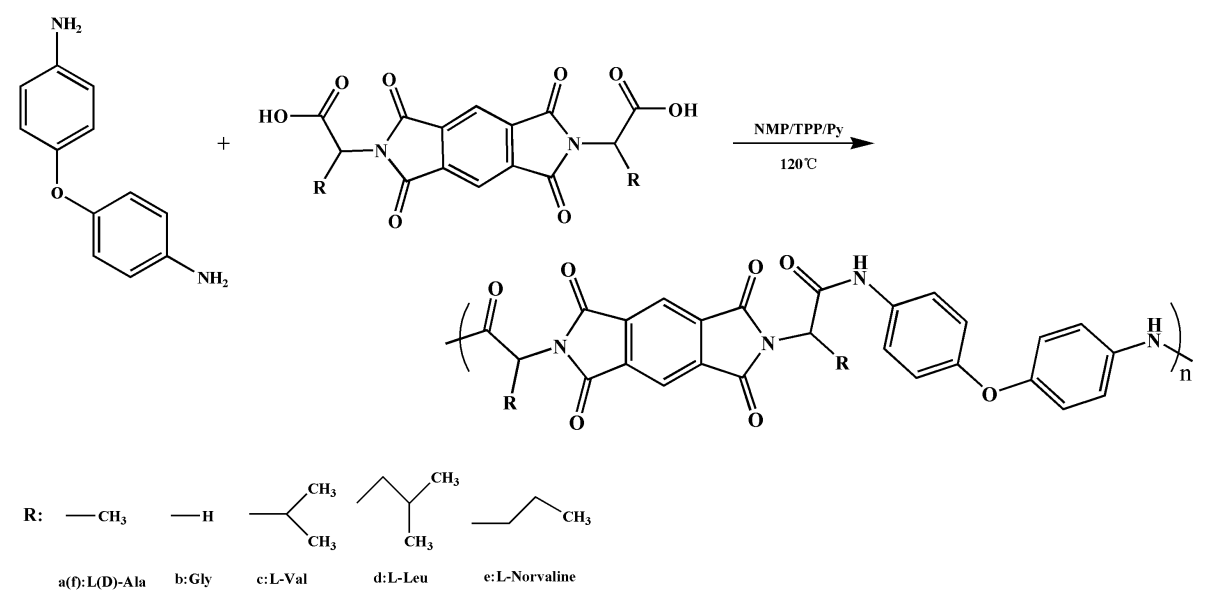

Scheme 2. Polymerization scheme of PAI3a-PAI3f.

\subsection{Preparation of PAI Films}

Firstly, $0.25 \mathrm{~g}$ of the prepared PAIs (PAI3a-PAI3f) was dissolved in $5 \mathrm{~mL}$ of DMF under sufficient stirring. After that, the solution was put into a Teflon mold with a diameter of $5 \mathrm{~cm}$. Finally, the pieces of clear flexible PAI films were obtained by vacuum drying at $50^{\circ} \mathrm{C}$.

\subsection{Enzymatic Degradation}

PAI3a-PAI3f films were placed with $10 \mathrm{~mL}$ of Tris- $\mathrm{HCl}$ buffer $(\mathrm{pH}=7.4)$ in the presence of proteinase $\mathrm{K}$ and $0.02 \%$ of sodium azide. Then each sample was placed in a constant temperature incubator shaker at $37 \pm 0.5{ }^{\circ} \mathrm{C}$ in order to restore the original level of enzymatic activity. The films were removed from the enzymatic solution every $24 \mathrm{~h}$, and washed with large quantities of ethanol and distilled water. Afterwards, they were dried under vacuum at $40{ }^{\circ} \mathrm{C}$ to a constant weight.

\section{Results and Discussion}

The structure of monomers2a-2f and PAI3a-PAI3f films were confirmed by IR and ${ }^{1} \mathrm{H}$ NMR spectra. The elemental analysis values of the polymers agreed well with the calculated values for the expected structures. (Figures S1-S4).

The thermal stability of PAI films was investigated through TGA and DSC. The TGA and DSC measurements of PAI3a-3f were explored, as shown in Figures S5 and S6, respectively. The glass 
transition temperature (Tg) obtained from DSC and the temperatures corresponding to a weight loss of $5 \%$ (T5) and 10\% (T10) of PAIs acquired from TGA are summarized in Table 1 . The temperatures of initial decomposition (T5 and T10) which ranged from $296^{\circ} \mathrm{C}$ to $323{ }^{\circ} \mathrm{C}$ and from $323^{\circ} \mathrm{C}$ to $390{ }^{\circ} \mathrm{C}$, respectively, and the high char yields $(46.15-58.67 \%)$ at $800^{\circ} \mathrm{C}$ of PAI3a-3f showed good thermal stabilities of these polymers.

Compared to PAI3f, PAI3a show a higher Tg value, which indicates that the PAIs with the L configuration have better thermal properties than those with the D configuration. In fact, we also investigated PAIs containing the other differential configurations of amino acids such as phenylalanine, leucine, etc. The PAIs with L configuration also have a higher Tg value. This may be the cause of natural selection. All these PAIs, except for the PAI3f, have the same polymer backbone, but have differential side groups based on differential amino acids. PAI3a and PAI3e have Tg values greater than PAI3b. The volume effect of the side group played a leading role, resulting in a higher Tg values for PAI3a and PAI3e. Among them, the side groups of PAI3a and PAI3e are all normal paraffin chains. The side groups of PAI3e have greater length and flexibility, resulting in internal plasticization. This makes the Tg value of PAI3e smaller than PAI3a. Through the comparison of PAI3b and PAI3c, a lower $\mathrm{Tg}$ value is attached to PAI3c. This may be due to the fact that the isolation effect dominated at the time, because the volume of the isopropyl group was much bigger than that of the methyl group. This increased the distance between the main chains and made it less likely to form hydrogen bonds, and also weakened the intermolecular forces. Therefore, PAI3c has the lowest Tg value. However, due to the combined effect of volume effect and isolation effect, PAI3d has a Tg value slightly lower than PAI3b and significantly higher than PAI3c. On one hand, in the polymer we synthesized, the L configuration and the D configuration of the amino acid affected the Tg values. On the other hand, when the main chain is the same, the volume effect of the side groups, the internal plasticizing effect and the isolation effect also had an effect on the Tg values.

Table 1. Thermal properties of PAI3a-3f.

\begin{tabular}{ccccc}
\hline Polymer & $\mathbf{T g}\left({ }^{\circ} \mathbf{C}\right)$ & $\mathbf{T}_{\mathbf{5}}\left({ }^{\circ} \mathbf{C}\right)^{\mathbf{a}}$ & $\mathbf{T}_{\mathbf{1 0}}\left({ }^{\circ} \mathbf{C}\right)^{\mathbf{b}}$ & Char Yield (\%) $^{\mathbf{c}}$ \\
\hline PAI3a & 241 & 313 & 354 & 58.67 \\
PAI3b & 196 & 323 & 390 & 48.90 \\
PAI3c & 147 & 278 & 323 & 46.15 \\
PAI3d & 189 & 297 & 340 & 47.25 \\
PAI3e & 206 & 296 & 329 & 47.86 \\
PAI3f & 238 & 310 & 357 & 56.15 \\
\hline
\end{tabular}

a Temperature at which $5 \%$ weight loss fully was recorded by TGA in a $\mathrm{N}_{2}$ atm. ${ }^{\mathrm{b}}$ Temperature at which $10 \%$ weight loss fully was recorded by TGA in a $\mathrm{N}_{2}$ atm. ${ }^{c}$ Weight percent of the material left undecomposed after TGA at $800{ }^{\circ} \mathrm{C}$ in a $\mathrm{N}_{2} \mathrm{~atm}$.

Figure 1 shows the change in molecular weight at different stages of enzymatic degradation. As the degradation time prolongs, the molecular weight gradually decreases. This illustrates the loss of small molecules during the degradation process. As can be seen in Figure 1, the fastest degradation rate of PAI3b is up to $50 \%$, PAI3e is the slowest degradation, and molecular weight loss is less than $10 \%$. Although glycine has no chirality, PAI3b degrades faster than PAI3a. This indicates that the structure of amino acids has the greatest influence on the degradation performance of PAI relative to chirality.

From PAI3a to PAI3c to PAI3d, the degradation rate gradually slows down. This indicates that for natural chiral amino acids, the rate of degradation decreases as the carbon chain grows. This may be because the hydrophilicity of polymers decreases with the increase of side-group carbon chains. The microorganisms in nature are all hydrophilic, so the longer the carbon chain of the side group, the more difficult it is to be degraded. PAI3e is an unnatural amino acid. Although its side-group carbon chains are shorter than the PAI3d, its degradation rate is the lowest.

As can be seen in Figure 1, PAI3a has the fastest degradation rate, higher than PAI3f, indicating that the microorganism preferentially degrades the PAIs of the L-configuration. This enantioselectivity 
stems from spin polarized interaction. This mechanism for the intermolecular interaction of chiral molecules results in a higher degradation rate of the PAI in the L-configuration [20,21]. When the structure of the amino acid introduced into PAIs is different, the effect of the structure on the degradation effect is more obvious. The degradation of natural chiral PAIs is better than that of non-natural chiral PAIs.

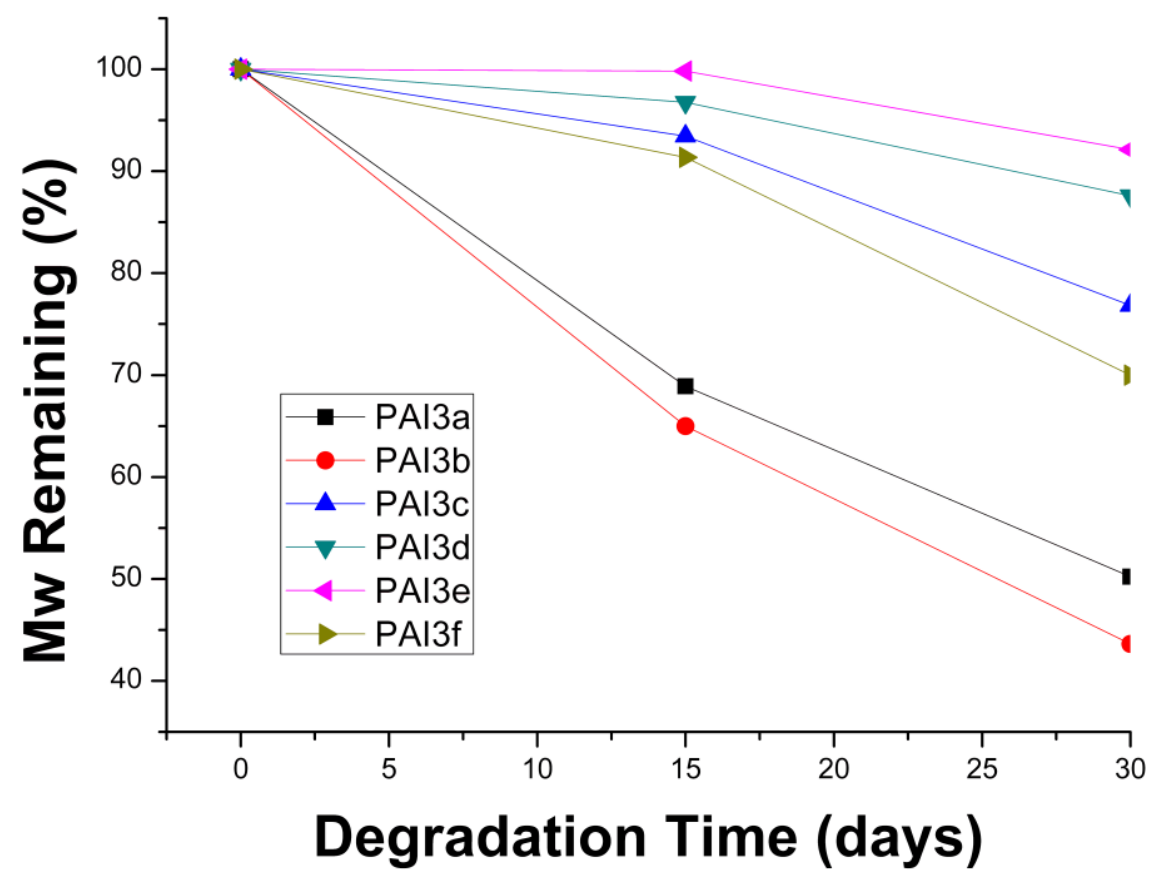

Figure 1. Weight average molecular remaining of PAI3a-PAI3f at each stage of degradation.

Figure 2 shows the infrared changes of PAI3a before and after degradation. It can be seen from Figure 2 that after 30 days of degradation, the symmetrical and asymmetric stretching vibration absorption bands of the carbonyl group at $1654 \mathrm{~cm}^{-1}$ and $1658 \mathrm{~cm}^{-1}$ are significantly weakened, and the bending vibration absorption band of $\mathrm{CN}$ at $1383 \mathrm{~cm}^{-1}$ is also significantly weakened. In addition, the bending vibration absorption band of $\mathrm{C}=\mathrm{O}$ at the $721 \mathrm{~cm}^{-1}$ of the imide five-membered ring almost disappeared, indicating that the carbonyl and imide bonds from the amino acid are easily destroyed during the degradation process. It can be found that the fragmentation of polymer chains occurs mainly on the two ends of amino acid molecules. It is to say that the amide and imide bonds sourced from carboxyl and amino groups in amino acids are easily broken. Figure 3 is a nuclear magnetic diagram of the degradation product of PAI3a. As can be seen from Figure 3, the main ${ }^{1} \mathrm{H}$ NMR peak of the degradation product is very similar to that of the polymer, containing benzene ring, methyl group, carboxyl group, amino group and other functional groups. This further indicates that the degradation of the PAI films begins with the broken of amide bonds, and later extends to the damages of imide rings. The break of polymer chain and the formation of small molecules leads to the change of molecular weight. 


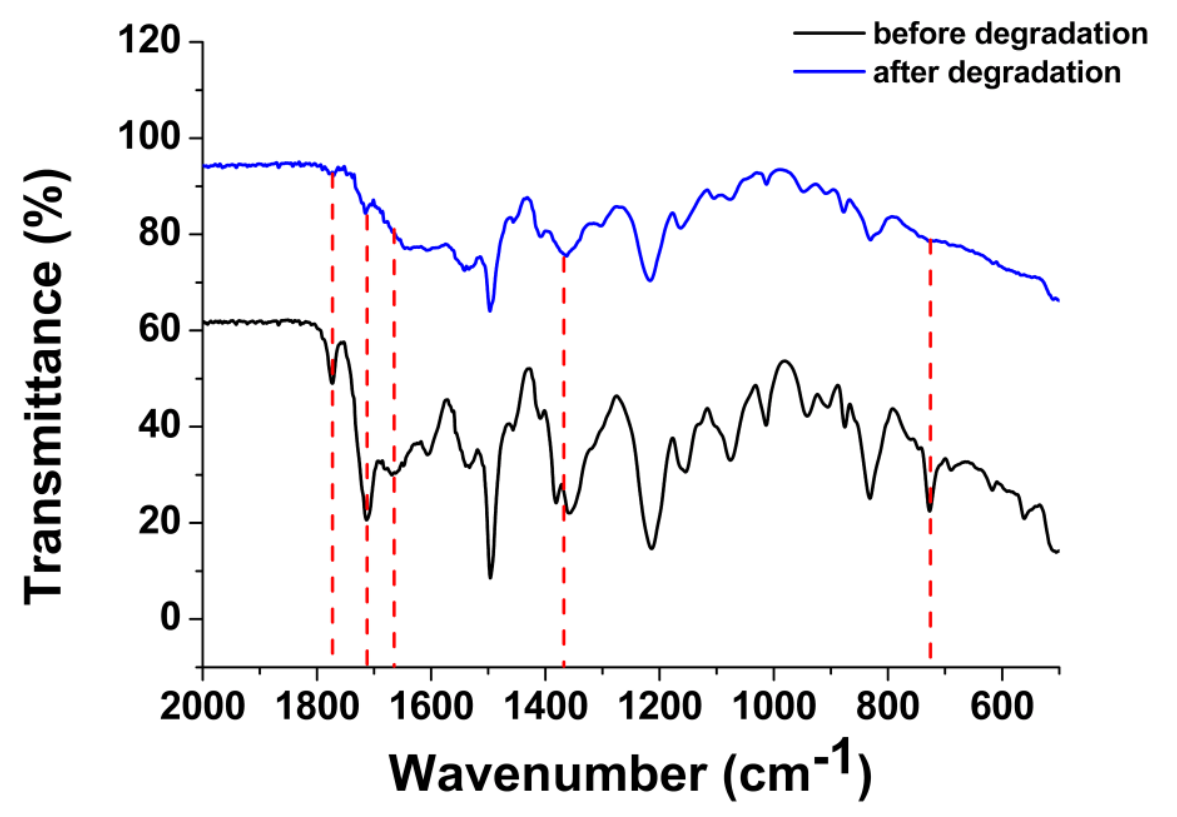

Figure 2. FTIR spectra of PAI3a film before and after degradation.

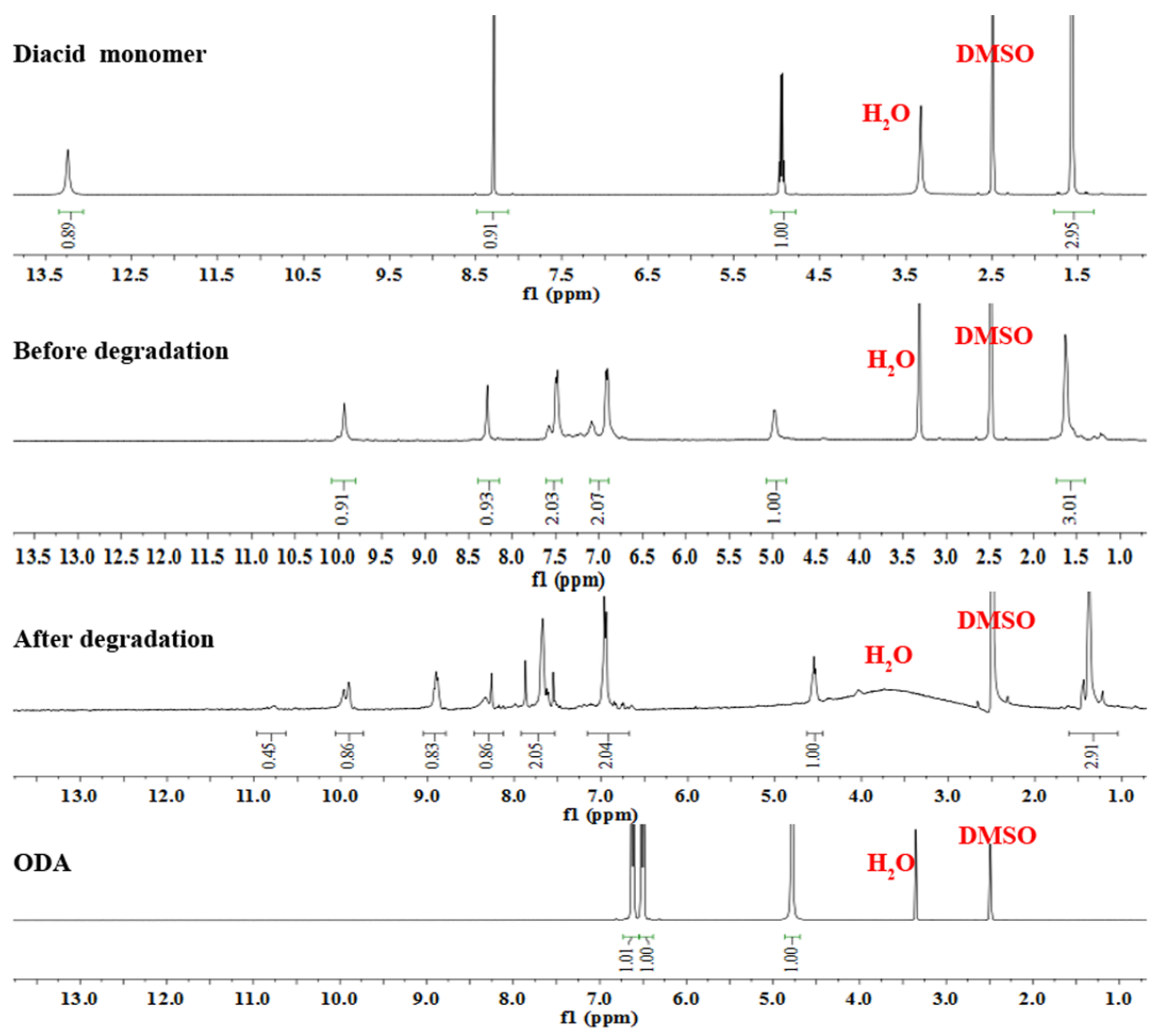

Figure 3. ${ }^{1} \mathrm{H}$ NMR spectra of PAI3a film before and after degradation.

X-ray diffractograms of PAI3b are shown in Figure 4. The other PAIs have similar X-ray diffractograms. PAI3b exhibited two broad halos in the range $2 \theta=20-25^{\circ}$ and $15-20^{\circ}$. The broad halos in XRD patterns indicates their amorphous nature [22]. Thus, the PAIs is completely amorphous. 


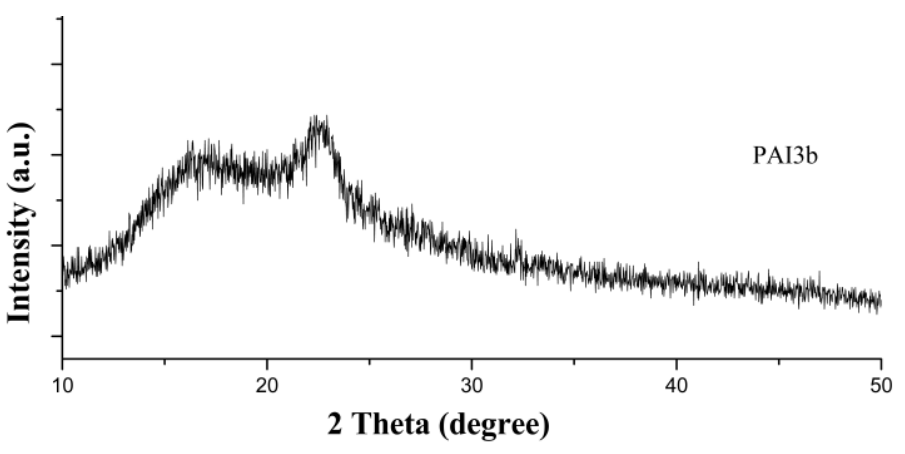

Figure 4. X-Ray diffractograms of PAI3b.

Figure 5 shows the SEM images of different periods of PAI3a and PAI3b degradation, and that the other PAIs have the similar change rules. It can clearly be seen from Figure 5 that after 15 days of degradation, there is a significant change in the surface of the membrane. The formation of pores has occurred. After 30 days of degradation, the damage of the membrane surface is more obvious, and there are more holes. This may be because the enzyme damages the PAIs membrane from the outside to the inside, first destroying the surface layer of the membrane, then gradually deepening, and finally forming holes.
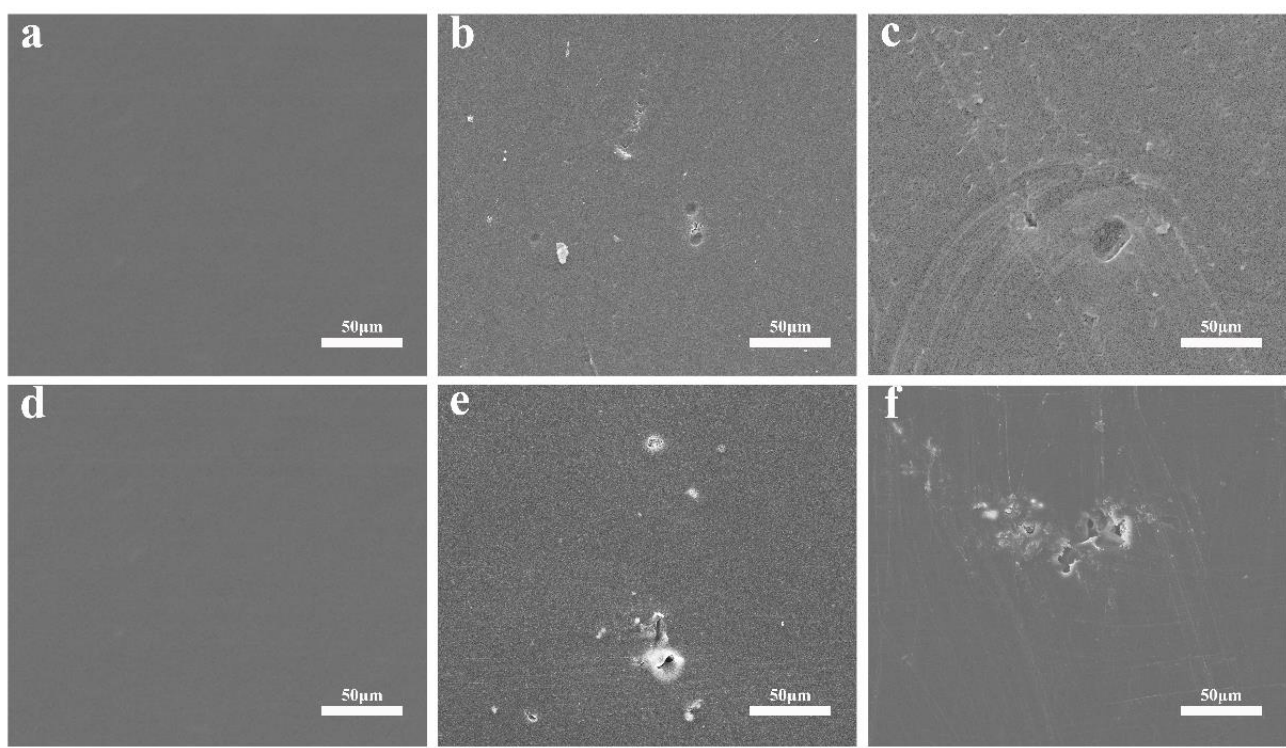

Figure 5. SEM images of PAI3a $(\mathbf{a}-\mathbf{c})$ and PAI3b $(\mathbf{d}-\mathbf{f})$ after each stage of degradation.

The water contact angle of the membrane surface at different degradation stages is shown in Figure 6. As can be seen from Figure 6, with the increase of degradation time, the water contact angle on the membrane surface becomes smaller and smaller, and also becomes more and more hydrophilic. This is because of the degradation of polymers, where polymer chains are broken and some hydrophilic functional groups are exposed to the surface of the membrane, which leads to the increase of hydrophilicity of the membrane. Polymers with simple pendant groups are more susceptible to degradation. The change in the water contact angle of PAI3b is more pronounced. Figure 5 shows that proteases can cause more damage to the backbone of the L-amino acid polymer than D-amino acids. More carboxyl and amino groups are exposed on the surface of the PAI film. Give PAI3a a higher surface hydrophilicity. The degradation of natural chiral PAIs is better than that of non-natural chiral PAIs. Thus, the PAI3d has a higher water contact angle. 


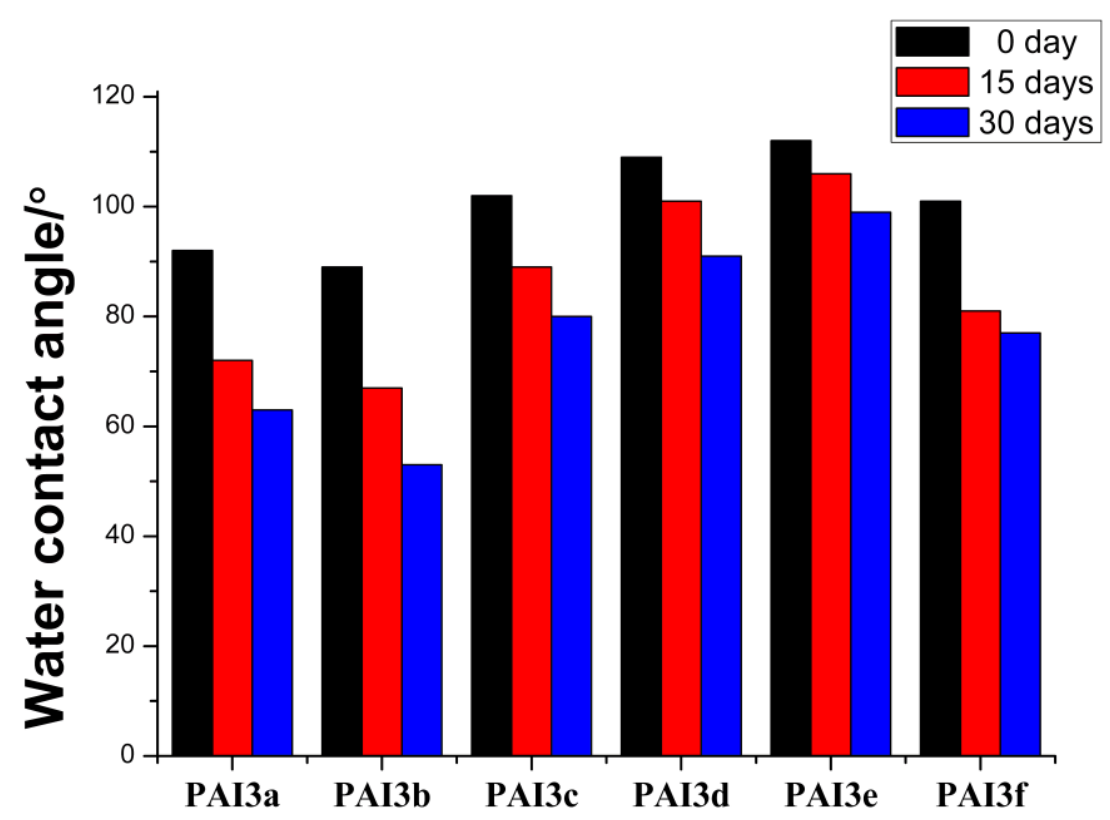

Figure 6. Water contact angles of PAI3a-PaI3f before and after degradation.

\section{Conclusions}

Thermal test shows the polymers (PAI3a-3f) have good thermal stability. PAIs with L configuration have higher $\mathrm{Tg}$ values than PAIs with D configuration. At the same time, the Tg values are also affected by the volume effect of the side groups, the internal plasticizing effect and the isolation effect. Water contact angle measurement that the polymer chains are broken and more carboxyl and amino groups are exposed on the surface of the PAI film, which leads to the increase of hydrophilicity of the membrane. The enzymatic degradation test suggests that the structure of amino acids have the greatest influence on the degradation performance of PAIs relative to chirality. For natural chiral amino acids, the rate of degradation decreases as the carbon chain grows. The degradation rate of natural chiral PAIs are higher than that of non-natural chiral PAIs. Degradation tends to be PAIs with simple side groups and L configurations. This makes it possible to develop polymer materials with specific degradationrates.

Supplementary Materials: The following are available online at http:/ /www.mdpi.com/2076-3417/9/3/578/s1. The following is the supplementary materials related to this article. Additional component analysis includes the yields and physical properties (see Table S1) of the diacid monomers(2a-2f), IR spectra (see Figure S1) and ${ }^{1} \mathrm{H}$ NMR spectra (see Figure S3) of the diacid monomers(2a-2f), the yields and physical properties (see Table S2) of the PAI3a-paI3f, IR spectra (see Figure S2) and ${ }^{1} \mathrm{H}$ NMR spectra (see Figure S4) of the PAI3a-3f, TGA graphs (see Figure S5) and DSC graphs (see Figure S6) of PAI3a-3f.

Author Contributions: Conceptualization, W.L.; Data curation, W.L., F.H., W.Y., Z.Y. and H.Z.; Formal analysis, W.L. and H.Z.; Funding acquisition, J.Y.; Methodology, W.L.; Writing—original draft, W.L.; Writing—review \& editing, J.Y.

Funding: This research was funded by the Shandong Provincial Natural Science Foundation, China, grant number ZR2012EMZ003.

Conflicts of Interest: The authors declare no conflict of interest.

\section{References}

1. Baillargeon, A.L.; Mequanint, K. Biodegradable polyphosphazene biomaterials for tissue engineering and delivery of therapeutics. Biomed. Res. Int. 2014, 2014, 761373. [CrossRef] [PubMed]

2. Andreas, L.; Robert, L. Biodegradable, elastic shape-memory polymers for potential biomedical applications. Science 2002, 296, 1673-1676. 
3. Thakur, V.K.; Thakur, M.K.; Raghavan, P.; Kessler, M.R. Progress in green polymer composites from lignin for multifunctional applications: A review. ACS Sustain. Chem. Eng. 2014, 2, 1072-1092. [CrossRef]

4. Siracusa, V.; Rocculi, P.; Romani, S.; Dalla Rosa, M. Biodegradable polymers for food packaging: A review. Trends Food Sci. Technol. 2008, 19, 634-643. [CrossRef]

5. Pilla, S.; Kramschuster, A.; Yang, L.; Lee, J.; Gong, S.; Turng, L.S. Microcellular injection-molding of polylactide with chain-extender. Mater.Sci. Eng. C 2009, 29, 1258-1265. [CrossRef]

6. Tachibana, Y.; Baba, T.; Kasuya, K.I. Environmental biodegradation control of polymers by cleavage of disulfide bonds. Polym. Degrad. Stabil. 2017, 137, 67-74. [CrossRef]

7. Faghihi, K.; Shabanian, M.; Valikhani, N. New poly(amide-imide)s based on 1,3-bis[4,4'-(trimellitimido) phenoxy] propane and hydantoin derivatives: Synthesis and properties. Des. Monomers Polym. 2011, 14, 109-119. [CrossRef]

8. Dodda, J.M.; Bělský, P. Progress in designing poly(amide imide)s (pai) in terms of chemical structure, preparation methods and processability. Eur. Polym. J. 2016, 84, 514-537. [CrossRef]

9. Mallakpour, S.; Iderli, M.; Sabzalian, M.R. In vitro studies on biodegradable chiral nanostructure poly(amide-imide)s containing different natural amino acids in green medium. Des. Monomers Polym. 2013, 16, 509-514. [CrossRef]

10. Zou, Q.; Zhou, Q.; Dai, H. Synthesis, mechanical properties and biocompatibility of novel biodegradable poly(amide-imide)s for spinal implant. Polym. Degrad. Stabil. 2017, 135, 85-98. [CrossRef]

11. Hazen, R.M.; Sholl, D.S. Chiral selection on inorganic crystalline surfaces. Nat. Mater. 2003, 2, 367-374. [CrossRef] [PubMed]

12. Ronald, B. Role of sulfur chirality in the chemical processes of biology. Chem. Soc. Rev. 2005, 34, 609-624.

13. Maier, N.M.; Franco, P.; Lindner, W. Separation of enantiomers: Needs, challenges, perspectives. J. Chromatogr. A 2001, 906, 3-33. [CrossRef]

14. Wang, X.; Gan, H.; Sun, T.; Su, B.; Fuchs, H.; Vestweber, D.; Butz, S. Stereochemistry triggered differential cell behaviours on chiral polymer surfaces. Soft Matter 2010, 6, 3851-3855. [CrossRef]

15. Hui, G.; Kangjian, T.; Taolei, S.; Michael, H.; Yong, L.; Lifeng, C.; Stefan, B.; Harald, F. Selective adsorption of DNA on chiral surfaces: Supercoiled or relaxed conformation. Angew. Chem. 2010, 48, 5282-5286.

16. Reeve, M.S.; McCarthy, S.P.; Downey, M.J.; Gross, R.A. Polylactide stereochemistry: Effect on enzymatic degradability. Macromolecules 1994, 27, 825-831. [CrossRef]

17. Gan, Z.; Abe, H.; Kurokawa, H.; Doi, Y. Solid-state microstructures, thermal properties, and crystallization of biodegradable poly(butylene succinate) (pbs) and its copolyesters. Biomacromolecules 2001, 2, 605. [CrossRef]

18. Li, P.; He, F.; Yang, Z.; Yang, W.; Yao, J. The degradability and thermal properties of chiral polyamide-imides synthesized from several L-amino acids: Side group effects. Polym. Degrad. Stabil. 2018, 147, 267-273. [CrossRef]

19. Wu, Q.; Yang, Z.; Yao, J.; Yu, D. Synthesis and biodegradation studies of optically active poly(amide-imide)s based on $N, N^{\prime}$-(pyromellitoyl)-bis-L-amino acid. High Perform. Polym. 2015, 28, 34-46. [CrossRef]

20. Kumar, A.; Capua, E.; Kesharwani, M.K.; Martin, J.M.; Sitbon, E.; Waldeck, D.H.; Naaman, R. Chirality-induced spin polarization places symmetry constraints on biomolecular interactions. Proc. Natl. Acad. Sci. USA 2017, 114, 2474-2478. [CrossRef]

21. Naaman, R.; Kumar, A.; Capua, E.; Vankayala, K.; Fontanesi, C. Magnetless device for conducting three-dimensional spin-specific electrochemistry experiments. Angew. Chem. 2017, 56, 14587-14590.

22. Kuhire, S.S.; Sharma, P.; Chakrabarty, S.; Wadgaonkar, P.P. Partially bio-based poly(amide imide)s by polycondensation of aromatic diacylhydrazides based on lignin-derived phenolic acids and aromatic dianhydrides: Synthesis, characterization, and computational studies. J. Polym. Sci. Part A Polym. Chem. 2017, 55, 3636-3645. [CrossRef]

(C) 2019 by the authors. Licensee MDPI, Basel, Switzerland. This article is an open access article distributed under the terms and conditions of the Creative Commons Attribution (CC BY) license (http:// creativecommons.org/licenses/by/4.0/). 